\title{
Société suisse de médecine du travail (SSMT)
}

\section{Brigitta Danuser, \\ Martin Rüegger}

\footnotetext{
* Depuis l'entrée en vigueur de la $5^{\mathrm{e}}$ révision de l'AI, ce type d'intervention devrait être soutenu par l'AI.
}

La Société suisse de médecine du travail est une petite société de 200 membres. Elle a pour objectif d'améliorer les conditions de travail là où elles sont nuisibles à la santé et de défendre les intérêts des médecins du travail sur le plan national.

\section{La capacité de travail au centre du débat}

Les questions concernant la capacité de travail intéressent de plus en plus les assurances sociales. La $5^{\mathrm{e}}$ révision de l'AI vise notamment la détection précoce d'une perte de la capacité de travail due à des raisons de santé afin de mieux pouvoir la maintenir. Cette démarche gagne en importance au vu du nombre de plus en plus important de travailleurs âgés et en prévision d'une augmentation de l'âge de la retraite.

Par capacité de travail, l'on entend les aptitudes biologiques, psychologiques et sociales dont dispose une personne pour répondre à une exigence de travail sans que sa santé ait à en pâtir. Par conséquent, la capacité de travail dépend non seulement de l'état de santé individuel de chaque personne, mais aussi des exigences du poste de travail.

\section{L'interaction entre l'individu et son travail: une compétence-clé de la médecine du travail}

Deux possibilités d'intervention majeures résultent de ce qui précède, à savoir les mesures individuelles (thérapie et formation des compétences) ainsi que l'amélioration des conditions du poste de travail. L'évaluation globale du rapport entre l'individu et son travail et la mise en évidence

Monsieur S., 49 ans, contrôleur qualité, $198 \mathrm{~cm}$, mince, est régulièrement mis en incapacité de travail par son médecin de famille en raison de douleurs à la nuque avec irradiation dans l'épaule droite.

L'examen par un médecin du travail a donné les résultats suivants:

douleurs non spécifiques dans la nuque liées au travail; limitations de la fonction de la ceinture scapulaire; musculature dorsale faible;

tendance à imaginer le pire;

sentiment de manque de reconnaissance par le supérieur hiérarchique;

souhait d'accomplir des tâches plus variées.

Résultats de l'analyse du poste travail effectuée en collaboration avec $\mathrm{M}$. S.:

- position assise: dos trop fortement penché vers l'avant sans appui dorsal. Tête constamment inclinée;

travail à l'écran: écran placé au moins $15 \mathrm{~cm}$ trop bas pour quelqu'un d'aussi grand;

capacité visuelle limitée face à l'écran et non corrigée. de mesures appropriées sont les compétences-clé de la médecine du travail. Autrement dit, promouvoir et maintenir la capacité de travail et faciliter la réinsertion de la personne, de même que la prévention et la détection précoce des maladies liées au travail font partie du domaine de compétences de la médecine du travail. Le cas présenté ci-après montre comment un médecin du travail peut, grâce à une évaluation globale, aider à fixer des objectifs thérapeutiques et améliorer les conditions de travail d'un patient.*

\section{Les ressources de la médecine du travail doivent être renforcées en Suisse}

Le manque de spécialistes dans ce domaine est un problème. Le plan d'action global («Global Plan of Action») sur le travail et la santé de l'OMS exige notamment que chaque travailleur puisse avoir accès à un service de médecine du travail. En Suisse, l'ordonnance 3 relative à la loi sur le travail précise que l'employeur doit mener une enquête relevant de la médecine du travail lorsque des éléments font apparaître que l'activité exercée par un travailleur porte atteinte à sa santé. C'est pourquoi la SSMT a pour but d'améliorer les ressources en médecine du travail en Suisse et de favoriser l'accès aux services de médecine du travail pour les personnes concernées. Pour y parvenir, il est nécessaire que les politiques soutiennent le savoir professionnel en médecine du travail et mettent à disposition des fonds et des postes de formation.

Mesures thérapeutiques définies en collaboration avec le médecin de famille:

- traitement ambulatoire à la demi-journée dans un centre de réadaptation avec pour objectif d'améliorer la fonctionnalité, de renforcer la musculature en général et d'acquérir les bases d'une position assise ergonomique; soutien psychologique et exercices de détente durant 6 semaines en vue de reprendre le travail le plus rapidement possible à $50 \%$;

correction de la vision;

adaptation du poste de travail: modification de la hauteur de l'écran et de la chaise;

- discussion avec le supérieur hiérarchique en vue de varier les tâches et d'aborder la question de la reconnaissance.

Deux mois après la reprise du travail, le domaine des tâches de M. S. a été élargi. Cela fait $\mathbf{1 4}$ mois qu'il n'a plus connu d'incapacité de travail. Les douleurs à la nuque, désormais sporadiques, sont supportables et la question de la reconnaissance s'est améliorée. 